\title{
PROCESS AND SYSTEMS A systems approach to trauma care in Myanmar: from health partnership to academic collaboration
}

\author{
Authors: Tom Bashford, ${ }^{\mathrm{A}}$ Phyu Phyu Nwe Myint, ${ }^{\mathrm{B}}$ Sein Win, ${ }^{\mathrm{C}}$ Myat Thu, ${ }^{\mathrm{D}}$ Mu Mu Naing, ${ }^{\mathrm{E}}$ Rowan Burnstein, ${ }^{\mathrm{F}}$

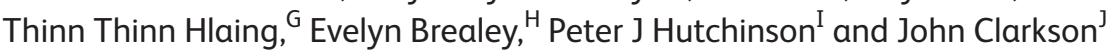

Experience from a variety of disciplines suggests that improving healthcare, particularly in resource-poor environments, can benefit from a systems approach. However, putting this into practice is challenging, especially in the context of an international institutional health partnership. In this article, we outline how a systems approach to the improvement of trauma care has informed both clinical improvement and academic collaboration as part of an ongoing partnership involving Cambridge University Hospitals NHS Foundation Trust, the University of Cambridge, and Cambridge Global Health Partnerships in the UK, and Yangon General Hospital, University of Medicine 1, and the Tropical Health and Education Trust (THET) in Myanmar. Improving and researching trauma care is an exemplar of a systems problem, requiring an understanding of the relevant people, equipment, processes, institutions, and power structures that result in the delivery of care at all points of the patient's journey from injury to rehabilitation. Exploring this in the explicit context of traumatic brain injury is one of the research themes of the NIHR Global Health Research Group on Neurotrauma, allowing systems research to directly inform efforts at practical improvement.

Authors: ${ }^{\text {A }}$ research fellow and anaesthetist, NIHR Global Health Research Group on Neurotrauma, University of Cambridge, UK; ${ }^{B}$ research fellow and anaesthetist, NIHR Global Health Research Group on Neurotrauma, University of Medicine 1, Myanmar; $C_{\text {research fellow and neurosurgeon, NIHR Global Health Research }}$ Group on Neurotrauma, University of Medicine 1, Myanmar; D professor of neurosurgery, NIHR Global Health Research Group on Neurotrauma, University of Medicine 1, Myanmar; Eprofessor of anaesthesia and intensive care, NIHR Global Health Research Group on Neurotrauma, University of Medicine 1, Myanmar; ${ }^{F}$ director, Cambridge-Yangon Trauma Intervention Partnership, Cambridge University Hospitals NHS Trust, UK; ${ }^{\mathrm{G}}$ THET Myanmar country director, Tropical Health and Education Trust, Myanmar; ${ }^{H}$ programme director, Cambridge Global Health Partnerships, Cambridge University Hospitals NHS Trust, UK; I professor of neurosurgery, NIHR Global Health Research Group on Neurotrauma, University of Cambridge, UK; ${ }^{3}$ professor of engineering design, Engineering Design Centre, University of Cambridge, UK

\section{Introduction}

Healthcare may be regarded as the product of a complex system of interactions between people, equipment, processes, and institutions. Efforts to improve care need to adopt a 'systems approach' to account for this complexity, with the World Health Organization (WHO) strongly endorsing systems thinking as a paradigm for healthcare improvement, both in terms of operational delivery and academic research. ${ }^{1}$ While systems thinking may be pertinent to improving any level of healthcare delivery, from national vaccination programmes to individual clinics, taking a systems approach to improvement can be challenging with comparatively few published examples and methodologies in the clinical literature. We describe a 5-year partnership between Cambridge University Hospitals NHS Foundation Trust (CUH), UK, and Yangon General Hospital (YGH), Myanmar as an example of an evolved systems approach to both improving and researching trauma care.

\section{The Cambridge-Yangon Trauma Intervention Project}

WHO figures and the Global Burden of Disease 2010 study suggest that trauma accounts for around $9 \%$ of deaths globally, or around 14,000 deaths per day, with the overwhelming majority of these occurring in low- and middle-income countries (LMICs). ${ }^{2,3}$ As a region, South East Asia shows the greatest burden of these injuries, both in terms of the current incidence and also the growth in the rate of injury. Myanmar has a population of 53 million people and health spending per capita of only \$20 USD, while the UK spends over $\$ 4,000$ USD. Current World Bank figures report a maternal mortality ratio (MMR) - often taken as a measure of a nation's health status - of 178 per 100,000 live births, as compared with the UK's 9 per 100,000 . It is estimated that $11 \%$ of all deaths in Myanmar are attributable to trauma. ${ }^{4-6}$

In 2013, CUH and YGH were awarded funding by the Tropical Health Education Trust (THET) to establish a health partnership with the aim of improving the care of trauma patients at YGH: the Cambridge-Yangon Trauma Intervention Project (CYTIP). This was conceived around the insight that care of the trauma patient requires a whole-system approach and involves multiple hospital teams: physiotherapy, nursing, pathology, surgery, and intensive care medicine (ICM). Interventions and exchange visits 
based around each of these areas were planned, with coordination by Cambridge Global Health Partnerships (CGHP) and THET Myanmar. A focus on a systems approach was carried through into the intervention design by these specific teams, as exemplified by the case of the ICM team which delivered two such interventions during the project.

\section{Delivering trauma intensive care (DelTICa) course: an educational intervention}

A 3-day course, 'Delivering trauma intensive care' (DelTICa), based around a systems approach to ICM, was iteratively developed between 2013 and 2017 with design input from UK and Myanmar clinicians. The aim of the course was to help delegates appreciate the value of the systems which underpin high-quality ICM care, and to change their own clinical practice within a systems framework. This course, redesigned over a number of iterations based on input from partners and participants, has taught a variety of clinicians including physicians, nurses, surgeons, and anesthetists, and is being expanded to other centres through further THET funding and with support from CGHP.

ICM exists within a system of systems: patients flow from the emergency department (ED) to the operating room (OR), to the intensive care unit (ICU), to the ward. Subsystems of blood transfusion, microbiology, nursing, physiotherapy, and infection control are vital, as are systems to ensure equipment and consumables are procured and maintained. The course is designed to examine how these can be coordinated and standardised, improving communication between stakeholders and strengthening clinical processes. Initial scoping work, formal and informal feedback after each iteration, ongoing discussion between partners, and independent monitoring and evaluation (Box 1), have all been incorporated into a cycle of redesign as the course has developed.

Short courses by high-income country partners in LMICs are at risk of advocating inappropriate models of care, and the course was designed instead to focus on the local development of practices through evidence-based frameworks such as care bundles. Even where the content is context-appropriate, courses can struggle to deliver sustainable behaviour change; to mitigate this the course has been integrated into the relevant local curricula, local trainers have been trained to deliver it

\section{Box 1.}

The qualitative and quantitative data show that the DeITICA Course has resulted in positive clinical change to the practice of intensive care in Yangon. The interviewees reported that they had gained both knowledge and skills through the course which had also provided them with practical methods of improving clinical care. Examples included an increase in hand-washing and awareness of infection control, the utilisation of care bundles and the systematic approach to the critical care patient. Interviewees related these examples to an improvement in patient care on the ward, including a reduction in infections and the ability to maintain a standard of care for all patients.

Monitoring and Evaluation Report: Cambridge Yangon Trauma Intervention Project

A Monkhouse Ltd 2017. independently, and local champions have taken on the mantle of driving clinical changes based on the course content. Embedding a short course within a long-term health partnership also helps promote improvement through mentoring, ongoing discussion, exchange visits, and mutual redesign of the course content. These principles are wholly consistent with the current experience of continuing professional development courses run as part of health partnership schemes. ${ }^{7}$

\section{A 10-year plan for intensive care medicine in Myanmar: an institutional intervention}

At the invitation of the rector of University of Medicine 1, Yangon, a nationwide survey of ICM capacity was completed by a joint UKMyanmar team to understand not only the human and material infrastructure available to deliver ICM in Myanmar, but also the clinical systems and processes which support ICM delivery. These data were then used to deliver a national workshop in partnership with the Ministry of Health and Sports in order to develop a 10-year plan for ICM in Myanmar.

There is overlap between ICM care and almost all elements of the acute care infrastructure both in terms of people, resources, and infrastructure. The existing academic literature on ICM provision, both in high- and low-income countries, was consulted to inform an assessment tool which was completed in all Myanmar intensive care units. The results of this survey were then presented to a national workshop of ICM stakeholders, convened by the Ministry of Health and Sports (MoHS), and used to facilitate an interactive discussion around the plan for ICM in Myanmar over the coming decade. Outputs from this workshop, along with the survey data, were captured and prepared into a report presented to the MoHS. A repeat survey is currently in the process of being planned, with a view to academic publication of the whole process as a joint UK-Myanmar project.

Traditional needs assessments often focus on human and material resources; however, simply describing a shortfall in these risks the inference that investment is the only way to improve care quality. This project seeks to understand not only the human and technical elements of ICM in Myanmar, but also the systems and processes in place to coordinate these elements. By using this combined information to inform the national workshop, the intention was to derive a roadmap for ICM in Myanmar which focused on developing a robust system, both to better marshal existing resources and support rational future investment.

\section{The NIHR Global Health Research Group on Neurotrauma}

The NIHR Global Health Research Group on Neurotrauma (GHRGN) at the University of Cambridge is a multinational partnership working to understand and improve the provision of care to patients experiencing neurotrauma (trauma to the brain and spinal cord) in LMICs. CGHP and THET are collaborators with the GHRGN on a joint research project seeking to develop the systems understanding gained through CYTIP, to model those systems which provide trauma care in Yangon with an emphasis on traumatic brain injury (TBI). ${ }^{8}$ This project, led by researchers in both the UK and Myanmar, aims to use narrative accounts from multiple stakeholders - including clinicians, patients, families, and managers - to elicit graphical models of care, using these to then plan clinical improvements across linguistic and cultural 


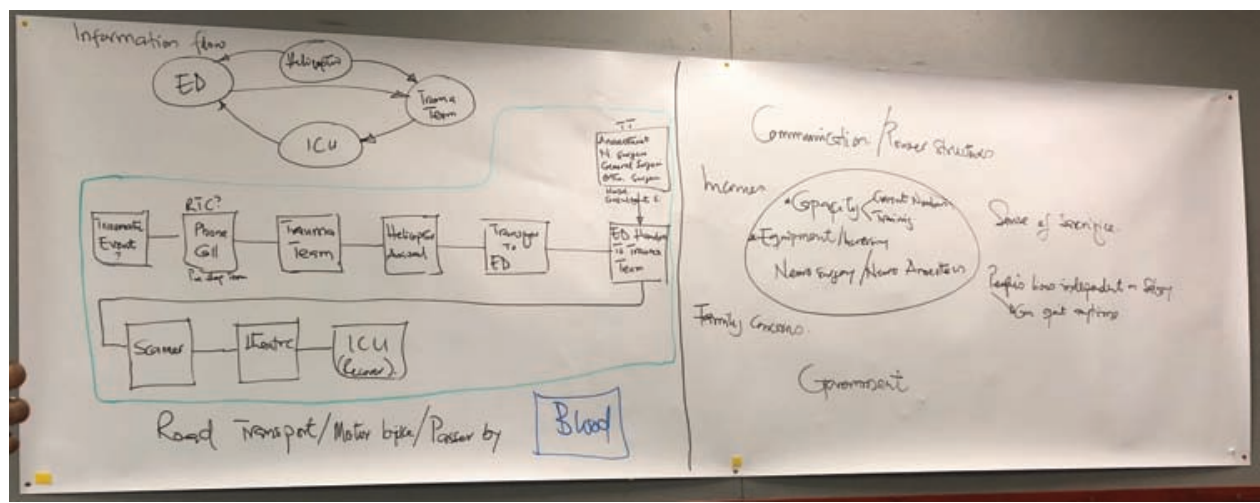

Fig 1. Process flow and information flow diagrams elicited from a UK case of traumatic brain injury (left) with influence diagram for current issues around TBI care in Myanmar (right). The diagrams represent an example of real-time system mapping in response to interview data, and were used to structure discussions around differences in communication between clinical staff, the role of the emergency department, and the problem of obtaining blood products for transfusion, among others. Each of these was identified as an example where systems in the UK and Myanmar operate differently, and where solutions to improve care might differ from the models used in the UK. (Photo ${ }^{\circ}$ The Authors).

barriers. A scoping workshop held in Cambridge for the research team has explored how narrative accounts can generate graphical systems maps, and how these can allow comparison of systems issues between environments (Fig 1). The aims of this ongoing project are twofold: to interrogate how a systems approach born out of UK experience translates to Myanmar, and to explore how systems mapping can facilitate improvement projects as part of an international healthcare partnership.

\section{Engineering better care}

Our experience has been that understanding systems of care in order to drive clinical improvement is challenging. 'Health systems' may describe national or regional building blocks of care delivery, or the complex sociotechnical system that delivers direct patient care. Similarly, 'improving systems' can mean focusing on material, human, or institutional resources, or on the interactions between these elements. While systems thinking is referenced within much of the quality improvement literature, applying this in practice can be daunting.

Approaches developed by the engineering community may assist clinical improvement efforts, particularly when trying to understand and augment complex systems of care. ${ }^{9,10}$ In 2017, these were highlighted in the report Engineering better care published by the UK's Royal Academy of Engineering, Academy of Medical Sciences, and Royal College of Physicians. ${ }^{11}$ The systems approach advocated in this report highlights people, systems, design and risk as fundamental areas to question when delivering clinical improvement, and also as areas of active research. While each area has a rich ontology and epistemology in its own right, the key message is that a balanced approach, based on the information and resources available at the time, is likely to yield the best results in engineering a new system of care. Furthermore, existing improvement methodologies may fail to address some of the key questions highlighted in this approach (Fig 2).

The four tenets of people, systems, design, and risk resonate well with the experience of CYTIP, and are informing the research work of the GHRGN in Myanmar. The ongoing human dialogue within the partnership has directly informed our shared view of the current systems of ICM care in Myanmar at both the local and national levels. This human-centred system view has in turn informed not only our intervention design, but also our understanding of the risks these interventions might pose and how they might be mitigated. The strength of the engineering paradigm in our setting is that it does not mandate complex methodology, but rather a balanced pragmatic approach in line with real-world experience.

\section{Conclusion}

The interface between academic theory and clinical improvement can be bewildering. The now-ubiquitous plan-do-study-act cycle, along with 'traditional' healthcare improvement methodologies can seem difficult to apply in complex systems, and indeed may fail to address key areas of a systems approach. The place for these approaches in low-resource environments is uncertain and informing 'real-world' improvement efforts with complicated theoretical frameworks can be very difficult. Beyond the published theory behind clinical quality improvement, there exists further levels of literature around health systems, trauma, neurotrauma, LMICs, and global surgery - all which are part of the context for the work of improving trauma care in Myanmar. ${ }^{12-17}$

Clinically, the challenge for CYTIP is to continue to identify areas for clinical improvement in the care of trauma patients in YGH and other partner hospitals, and to design and deliver appropriate interventions to address these. Academically, the challenge for the GHRGN is to develop the systems mapping methodology in both new clinical contexts and new countries. While much work has been done on systems research, many of the tools developed have not been extensively used in LMICs where issues of language, culture, power, and resources may all exert an influence on a methodology developed elsewhere. Work is needed to understand how models of care can be discussed in language-neutral and solution-neutral terms, to allow mutual 'problem architecturing'. The essence of partnership is co-creation and this requires both academic methodology and real-world practicality. The success of CYTIP and the GHRGN will be in the marrying of these within a common framework. 


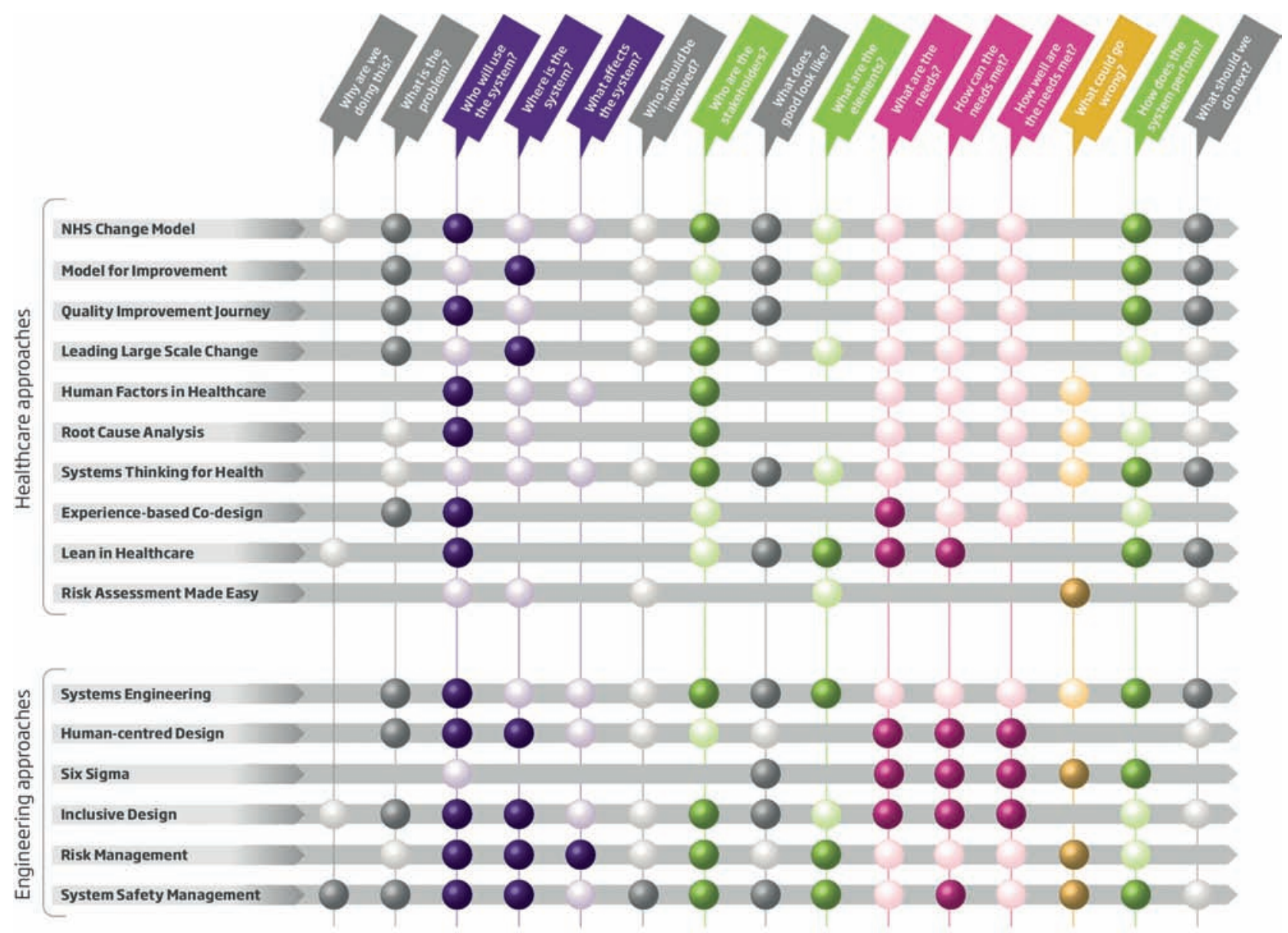

Clear guidance and tools

Description of approach only

Fig 2. A systems approach - presented as a series of ordered questions around general improvement (grey), people (purple), systems (green), design (pink), and risk (orange) - mapped against both traditional and engineering techniques for improvement. Reproduced with permission from Engineering Better Care (Royal Academy of Engineering, Academy of Medical Sciences and Royal College of Physicians, 2017).

\section{Conflicts of interests}

PJC is a fellow of the Royal Academy of Engineering and lead author of the cited report Engineering better care: a systems approach to health and care design and continuous improvement.

\section{Author contributions}

TB was responsible for drafting the original manuscript and all other authors reviewed and amended the manuscript before submission. All authors had an opportunity to review the final manuscript before submission, although TB takes overall responsibility for the content. The views expressed in this publication are those of the author(s) and not necessarily those of the NHS, National Institute for Health Research or the Department of Health.

\section{Ethical approval}

The workshop titled 'Developing a systems approach to traumatic brain injury in Myanmar' was submitted to the Research Ethics Committee of the Department of Engineering, University of Cambridge and given approval with respect to ethical considerations, 13 March 2018

\section{Acknowledgements}

This work was funded by the National Institute for Health Research (NIHR) Global Health Research Group on Neurotrauma using Official Development Assistance (ODA) funding from the UK Government. The Cambridge Yangon Trauma Intervention Partnership was supported by the Tropical Health Education Trust's (THET) Health Partnership Scheme, funded through the UK Department for International Development (DFID). PJH is funded by a NIHR Research Professorship and the NIHR Cambridge BRC. 


\section{References}

1 World Health Organization. Everybody's business: strengthening health systems to improve health outcomes: WHO's framework for action. WHO, 2007.

2 Sakran JV, Greer SE, Werlin E, McCunn M. Care of the injured worldwide: trauma still the neglected disease of modern society. Scand J Trauma Resusc Emerg Med 2012;20:64.

3 Stewart B, Khanduri P, McCord C et al. Global disease burden of conditions requiring emergency surgery. Br J Surg 2014;101:922.

4 Myanmar Ministry of Health and Sport. Myanmar National Health Plan 2017-2021. MoHS, 2016.

5 Latt NN, Myat Cho S, Htun NM et al. Healthcare in Myanmar. Nagoya J Med Sci 2016;78:123-34.

6 World Health Organization. The Republic of the Union of Myanmar health system review. Manila: WHO Regional Office for the Western Pacific, 2014.

7 Kudsk-Iversen S, Shamambo N, Bould MD. Strengthening the anesthesia workforce in low- and middle-income countries. Anesth Analg 2018;126:1291-7.

8 Bashford T, Clarkson PJ, Menon DK, Hutchinson P JA. Unpicking the Gordian knot: a systems approach to traumatic brain injury care in low-income and middle-income countries. BMJ Glob Heal 2018;3:e000768.

9 National Academy of Engineering (US) and Institute of Medicine (US) Committee on Engineering and the Health Care System; Reid PP, Compton WD, Grossman JH et al (eds). Building a Better Delivery System: A New Engineering/Health Care Partnership. Washington, DC: National Academies Press (US), 2005.

10 Watts BV, Shiner B, Ceyhan ME et al. Health Systems engineering as an improvement strategy: a case example using location-allocation modeling. J Healthc Qual 2013;35:35-40.
11 Clarkson J, Bogle D, Dean D et al. Engineering Better Care. A Systems Approach to Health and Care Design and Continuous Improvement. Royal Academy of Engineering, 2017.

12 Maas AIR, Menon DK, Adelson PD et al. Traumatic brain injury: integrated approaches to improve prevention, clinical care, and research. Lancet Neurol 2017:4422:987-1048.

13 Meara JG, Leather AJ, Hagander L et al. Global Surgery 2030: Evidence and solutions for achieving health, welfare, and economic development. Lancet 2015;386:569-624.

14 Rubiano AM, Carney N, Chesnut R, Puyana JC. Global neurotrauma research challenges and opportunities. Nature 2015;527:S193-7.

15 Carey G, Malbon E, Carey N et al. Systems science and systems thinking for public health: a systematic review of the field. BMJ Open 2015;5:e009002.

16 Adam T, de Savigny D. Systems thinking for strengthening health systems in LMICs: need for a paradigm shift. Health Policy Plan 2012;27(Suppl 4):iv1-3.

17 Adam T, Hsu J, de Savigny D et al. Evaluating health systems strengthening interventions in low-income and middle-income countries: are we asking the right questions? Health Policy Plan 2012;27(Suppl 4):iv9-19.

Address for correspondence: Dr Tom Bashford, NIHR Global Health Group on Neurotrauma, Division of Anaesthesia, University of Cambridge, Box 93, Addenbrooke's Hospital, Hills Road, Cambridge CB2 0QQ, UK.

Email: tb508@cam.ac.uk 